\title{
Investigation of a new feature for angular defect quantification by eddy current pulsed thermography
}

\author{
Junzhen Zhu ${ }^{1}$, Guiyun Tian ${ }^{1,2 *}$, Wenbo Duan ${ }^{3}$ \\ ${ }^{1}$ School of Engineering, Newcastle University, Newcastle upon Tyne NE1 7RU, UK \\ ${ }^{2}$ School of Automation Engineering, University of Electronic Science and Technology of China, Chengdu, China \\ ${ }^{3}$ Department of Mechanical, Aerospace and Civil Engineering, Brunel University London, Uxbridge, Middlesex UB8 \\ 3PH, UK g.y.tian@uestc.edu.cn; g.y.tian@ncl.ac.uk
}

\begin{abstract}
Eddy current pulsed thermography (ECPT) as one of the emerging NDT\&E techniques has been used for the evaluation of the integrity of rail tracks, especially for rolling contact fatigue (RCF) or angular defect detection and quantification. This paper proposed a new feature for angular defect quantification. Specifically, the first order derivative of the transient thermal response was calculated. Then a new feature derived from the first order derivative result was used to quantify the length of the angular slot. R-squared value and 2-norm of the residual were used to evaluate and compare the fitted linear relationship between different extracted features and the angular slot lengths. Through the comparison with other features, the strength and limitation of this new feature were summarized.
\end{abstract}

